\title{
Personhood: an inconvenient truth
}

\author{
Lodovico Balducci
}

Received: 31 October 2012/Accepted: 31 October 2012/Published online: 23 November 2012

(C) Springer-Verlag Italia 2012

The ultimate goal of medicine is the service of the person. This axiom, implied in the Hippocratic Oath, assumes that life, health, and freedom from discomfort are preconditions for the full expression of personal riches. All cultures seem to subscribe to this axiom, irrespective of religious beliefs or social and political organizations, situation in which one may consult a physician with the goal of "getting worse" in terms of health or of symptoms.

Surprisingly, the references to the person are scarce and inconsistent in the medical literature. Somehow the medical literature, that is, the medical establishment appears to skirt the issue on the tacit assumption that consensus exists on personhood and that any new exploration of this construct is unnecessary. Somehow the medical industrial establishment may feel threatened by a deeper exploration of personhood, on the fear that renewed awareness of personhood may threaten current medical practices as well as the conduct of medical research.

The principles of bioethics, the discipline charged with protecting the person in medical practice and medical research, include autonomy, beneficence, absence of maleficence, and justice [1]. They provide the benchmark for judging the legitimacy of medical interventions and of human investigations. Yet, controversy exists as to whom or what they do apply. Purposefully I mention "whom" and "what." The questions "who is a person" or "what is a person" are the two poles of the battery energizing all ethical debates. They entail two different constructs of the person. "Who is a person?" implies that every human life is endowed with personhood; "what is a person?" implies

L. Balducci $(\square)$

Senior Adult Oncology Program, Moffitt Cancer Center,

Tampa, FL, USA

e-mail: lodovico.balducci@moffitt.org that personhood pertains only to selected human lives expressing some characteristics that are objectively identifiable [2]. These generally include the capacity of selfdetermination.

The debate over personhood is further complicated by the current call for "personalized" medicine which refers to the individual characteristics of the disease rather than of the patient [3]. For example, personalized oncology refers to the identification of patients who may benefit from a specific treatment according to the tumor genomic or proteomic profile [4].

Perhaps a recent symposium supported by the King's College center for Humanities and Health [5] represents the most important endeavor in the attempt to define personhood in medical practice. This consensus conference included medical professionals as well as philosophers from around the world and was aimed to provide references to define "disorders of identity." While it afforded important clinical insights into identity, that is, a critical component of personhood, the conference fell short of answering the questions "who is a person" and "what is a person."

Personhood is frequently mentioned in two other medical areas. One involves the debate related to the beginning and the end of life [6]. In this case the definition of personhood is invoked by opposite parties to legitimize or to condemn abortion, euthanasia, and physician assisted suicide. The other involves the preservation of personhood as a form of palliative care for individuals affected by incurable and terminal diseases [7]. This novel approach to personhood is germane to the inspiration of the Journal of Medicine and the Person. The main goal of our publication and our movement is indeed to improve medical care by emphasizing and utilizing the patient's personal endowments.

Properly in my opinion, this issue of the Journal explores the construct of personhood in medicine. Without 
pretending to answer all the questions related to personhood, we hope to have laid the foundation for additional studies and to have provided a forum for a constructive debate involving all medical professionals. Mostly, we wish to rekindle the interest in the person as the ultimate beneficiary of medical intervention and scientific progress. More than condemning medical practices inconsistent with personal dignity, our goal is to enable the health professionals to exploit the different personal resources of each patient in the provision of personal care. Personal care is threatened by a medicine that has minimized the opportunities of human interactions. While nobody should dispute the benefits of modern technology, nobody should ignore what George Bernanos has prophesized 70 years ago in the book "La France contre le robots:" "Freedom is the first casualty in a world won over by technology." Our goal is to harness the forces of technology to the service of the person and to prevent them from acquiring a life on their on.

The four articles included in this issue have been selected in order of providing the reader with an outline of the major issues related to personhood in medicine. Dr. Shah reviews the evolving concepts of personhood since the beginning of the times and its application to medical activities. Dr. Spagnolo contrasts the two predominant schools of thoughts related to personhood in medical ethics. Drs. Donati and De Gaetano explore the role of personhood in medical research; drawing from their own experience this remarkable couple of scientists, who have been married to each other for almost half a century, underline how the predominance of the person has permeated their whole research experience. Not only have they endeavored to reach an unsurpassed expertise in their field, but also to share this experience by mentoring younger generations of scientists and to conduct research that is meaningful and honest. Equally important has been to create an environment in which each person, including the administrative and technical personnel felt motivated to give the best of themselves, because recognized their own role as essential. Finally, Dr. Tullis provides an analysis of personhood at the end of life. The goal of her paper is twofold. In addition to illustrating how awareness of personhood is indispensable to effective palliative and end-of life care, she proposes a novel and promising method to study personhood with qualitative research. Based on open end interviews, content and language analysis, qualitative research may enlarge the scope of biomedical research and allow the introduction of dimensions that are not less real for the fact that are not measurable.

We are the Journal of medicine and the Person. This issue of the journal illustrates who we are and why we are here.

Conflict of interest None.

\section{References}

1. Schmid-Felzman H (2003) Pragmatic principles: methodological pragmatism in the principle-based approach to bioethics. J Med Philos 28:581-596

2. Spagnolo AG (2012) Personhood: order and border of bioethics. J Med Pers. doi:10.1007/s12682-012-0135-9

3. Basik M, Balducci L, Bregni M (2011) Personalized medicine: a comprehensive approach in Oncology. J Med Pers 9:89-90

4. Pinto N (2012) Pharmacogenomic in Cancer care: adding some science to the art of medicine. Pers Med Oncol 1:56-61

5. Kingma E, McCabe MM (2012) Interdisciplinary workshop report: methodology and personhood and identity in medicine. J Eval Clin Pract 18:1057-1063

6. Cox DRA (2011) The problems with utilitarian conceptions of personhood in the abortion debate. J Med Ethics 37:318-320

7. Passmore MJ, Ho A, Gallagher R (2011) Behavioral and psychological symptoms in moderate to severe Alzheimer's disease: a palliative care approach emphasizing recognition of personhood and preservation of dignity. J Alzheimer's Dis 29:1-13 